\title{
RACSAMI
}

Rev. R. Acad. Cien. Serie A. Mat.

VOL. $103(1), 2009$, pp. 1-9

Análisis Matemático / Mathematical Analysis

\section{Holomorphically Dependent Generalised Inverses}

\author{
Seán Dineen and Milena Venkova
}

\begin{abstract}
In this article we investigate when the pointwise existence of a generalised inverse for holomorphic operator-valued mappings defined on domains in a Banach space implies the existence of a holomorphic generalised inverse.
\end{abstract}

\section{Inversas Generalizadas Holomórficamente Dependientes}

Resumen. En este artículo investigamos cuándo la existencia puntual de una inversa generalizada de una aplicación holomorfa operador-valuada definida en un dominio de un espacio de Banach implica la existencia de una inversa generalizada holomorfa.

\section{Introduction}

Let $f$ denote a holomorphic mapping from a domain $\Omega$ in a Banach space into $\mathcal{L}(X, Y)$, the space of continuous linear mappings from the Banach space $X$ into the Banach space $Y$. Over many years different authors, e.g. [1, 2, 4, 5, 7, 12], have considered when pointwise invertibility properties, of various kinds, imply the existence of a globally smooth inverse of the same kind. For example, if $f(z)$ has a right inverse for each $z \in \Omega$ does there exist $g$, holomorphic on $\Omega$ with values in $\mathcal{L}(Y, X)$, such that $g(z)$ is a right inverse for a $f(z)$ for all $z \in \Omega$ ? In this paper we continue our investigations of such problems. Many results are known when $\Omega$ is a domain in a finite dimensional space and our interest is focused on the problems that arise when $\Omega$ is a domain in an infinite dimensional space.

We refer to [6, 10] for background information on operators between Banach spaces, to [3, 9] for the theory of holomorphic mappings on Banach spaces and to [6, 7, 12] for classical results on holomorphic dependence of operator-valued functions over finite dimensional complex manifolds.

\section{Linear Preliminaries}

If $X$ and $Y$ are Banach spaces over $\mathbb{C}, \mathcal{L}(X, Y)$ will denote the space of all continuous linear operators from $X$ to $Y$ and $G L(X, Y)$ will denote the set of all invertible linear operators from $X$ to $Y$. If $X$ and $Y$ are subspaces of the Banach space $Z$ we use the notation $Z=X \oplus Y$ to indicate that $X$ and $Y$ are closed complemented subspaces of $Z$ and that $Z$ is the direct sum of $X$ and $Y$. We let $\mathcal{H}(\Omega, X)$ denote the set of all $X$-valued holomorphic mappings defined on an open subset $\Omega$ of a Banach space. We also use the standard notation $\mathcal{L}(X):=\mathcal{L}(X, X)$ and $G L(X):=G L(X, X)$.

Presentado por / Submitted by Amable Liñán Martínez

Recibido / Received: 11 de octubre de 2009. Aceptado / Accepted: 14 de enero de 2008.

Palabras clave / Keywords: Generalised inverse, complemented subspace, vector bundle, unconditional basis, projection.

Mathematics Subject Classifications: 46G20

(C) 2009 Real Academia de Ciencias, España. 
Definition 1. Let $T \in \mathcal{L}(X, Y)$. If $S \in \mathcal{L}(Y, X)$ and $T S T=T$ we call $S$ a pseudo-inverse for $T$. If, in addition, $S T S=S$ we call $S$ a generalised inverse for $T$. If $T S=\mathbf{1}_{Y}$ we call $S$ a right inverse for $T$. The operator $T$ is called splitting if $\operatorname{ker}(T)$ and $\operatorname{im}(T)$ are complemented in $X$ and $Y$ respectively.

The following proposition contains some important known results about generalised inverses ([2, 12]).

Proposition 1. If $X$ and $Y$ are Banach spaces and $T \in \mathcal{L}(X, Y)$ then the following are equivalent:

(a) Thas a pseudo-inverse,

(b) Thas a generalised inverse,

(c) $T$ is a splitting operator.

Right inverses are generalised inverses and generalised inverses are pseudo-inverses. If $S$ is a pseudoinverse for $T$ then STS is a generalised inverse for $T$.

We require the following construction of a generalised inverse. Let $T \in \mathcal{L}(X, Y)$ and suppose $X=$ $\operatorname{ker}(T) \oplus X_{1}$ and $Y=Y_{1} \oplus \operatorname{im}(T)$ are direct sum decompositions. The restriction of $T$ to $X_{1}, T_{R}$, is a continuous bijective linear mapping from $X_{1}$ onto $\mathrm{im}(T)$ and has, by the open mapping theorem, a continuous inverse, $T_{R}^{-1}$. We define $S: Y \rightarrow X$ by letting $S\left(y_{1}+y_{2}\right)=T_{R}^{-1}\left(y_{2}\right)$ for $y_{1} \in Y_{1}$ and $y_{2} \in \operatorname{im}(T)$. If $x_{1} \in \operatorname{ker}(T)$ and $x_{2} \in X_{1}$ then

$$
T S T\left(x_{1}+x_{2}\right)=T S T\left(x_{2}\right)=T\left(T_{R}^{-1} T\left(x_{2}\right)\right)=T\left(x_{2}\right)=T\left(x_{1}+x_{2}\right)
$$

and $T S T=T$. Moreover, if $y_{1} \in Y_{1}$ and $y_{2} \in \operatorname{im}(T)$, then

$$
S T S\left(y_{1}+y_{2}\right)=S\left(T T_{R}^{-1}\left(y_{2}\right)\right)=S\left(y_{2}\right)=S\left(y_{1}+y_{2}\right),
$$

and $S$ is a generalised inverse for $T$.

Lemma 1. If $P$ and $Q$ are projections in $\mathcal{L}(X)$ and $\|P-Q\|<1$ then $\left(\mathbf{1}_{X}-P+Q\right) \in G L(X)$ and $\left(\mathbf{1}_{X}-P+Q\right)(P(X))=Q(X)$. In particular, $P(X) \simeq Q(X)$.

Proof. Let $R:=\mathbf{1}_{X}-P+Q$. Since $\left(\mathbf{1}_{X}-P+Q\right) P=Q P$ we have

$$
R(P(X))=\left(\mathbf{1}_{X}-P+Q\right)(P(X)) \subseteq Q(X) .
$$

Since $\|P-Q\|<1, R:=\mathbf{1}_{X}-P+Q \in G L(X)$ and

$$
R^{-1}=\left(\mathbf{1}_{X}-P+Q\right)^{-1}=\sum_{n=0}^{\infty}(P-Q)^{n}=\left[\sum_{n=0}^{\infty}(P-Q)^{2 n}\right]\left(\mathbf{1}_{X}+P-Q\right) .
$$

Interchanging $P$ and $Q$ in (1) we obtain $\left(\mathbf{1}_{X}-Q+P\right)(Q(X)) \subseteq P(X)$ and as $(P-Q)^{2} P=P\left(\mathbf{1}_{X}-Q P\right)$ we see that $(P-Q)^{2} P(X) \subseteq P(X)$. Hence $R^{-1}(Q(X)) \subseteq P(X)$ and $Q(X) \subseteq R(P(X))$. Combining this with (1) completes the proof.

\section{Vector Bundles}

In this section we recall the definition of holomorphic Banach vector bundles and generalise to Banach spaces a result of Shubin [11] (see also [12, Theorem 3.11]).

Let $\pi: \mathcal{E} \rightarrow \Omega$ be a surjective holomorphic map of complex Banach manifolds. We assume that the fibre above $z \in \Omega, \mathcal{E}_{z}:=\pi^{-1}(z)$, has been given a Banach space structure whose topology coincides with the topology induced from $\mathcal{E}$. A collection $\left(U_{\alpha}, \tau_{\alpha}\right)_{\alpha \in \Lambda}$ is called a trivialising cover for $\pi$ if $\left(U_{\alpha}\right)_{\alpha \in \Lambda}$ is an open cover of $\Omega$ and for each $\alpha \in \Lambda$ there is a Banach space $X_{\alpha}$ such that $\tau_{\alpha}: \pi^{-1}\left(U_{\alpha}\right) \rightarrow U_{\alpha} \times X_{\alpha}$ is a biholomorphic mapping and conditions (i), (ii) and (iii) below are satisfied. 
(i) $\tau_{\alpha, z}:=\left.\tau_{\alpha}\right|_{\mathcal{E}_{z}}$ is a linear isomorphism ${ }^{1}$, from $\mathcal{E}_{z}$ onto $X_{\alpha}$ for each $z \in U_{\alpha}$.

(ii) $\left.\pi\right|_{\pi^{-1}\left(U_{\alpha}\right)}=\pi_{\alpha} \circ \tau_{\alpha}$, where $\pi_{\alpha}$ is the canonical projection from $U_{\alpha} \times X_{\alpha}$ onto $U_{\alpha}$. Conditions (i) and (ii) imply that $\rho_{\alpha \beta}:=\left.\tau_{\alpha} \circ \tau_{\beta}^{-1}\right|_{U_{\alpha \beta} \times X_{\beta}}$ has the form $\rho_{\alpha \beta}(z, x)=\left(z, g_{\alpha \beta}(z) x\right)$, where $g_{\alpha \beta}(z) \in \mathcal{L}\left(X_{\beta}, X_{\alpha}\right)$ and $x \in X_{\beta}$ whenever $\alpha, \beta \in \Lambda$ and $z \in U_{\alpha \beta}:=U_{\alpha} \cap U_{\beta} \neq \emptyset$.

(iii) If $\alpha, \beta \in \Lambda$ and $U_{\alpha} \cap U_{\beta} \neq \emptyset$ then the map $z \mapsto g_{\alpha \beta}(z)$ from $U_{\alpha \beta}$ into $\mathcal{L}\left(X_{\beta}, X_{\alpha}\right)$ is holomorphic.

Two trivialising covers are said to be equivalent if their union is also a trivialising cover.

Definition 2. A holomorphic vector bundle is a triple $(\mathcal{E}, \pi, \Omega)$, where $\pi: \mathcal{E} \rightarrow \Omega$ is a surjective holomorphic map of complex Banach manifolds, together with a class of equivalent trivialising covers for $\pi$.

We call $\mathcal{E}$ the bundle space, $\pi$ the projection of the bundle, $\Omega$ the base of the bundle, $\left\{\tau_{\alpha}: \pi^{-1}\left(U_{\alpha}\right) \rightarrow\right.$ $\left.U_{\alpha} \times X_{\alpha}\right\},\left(U_{\alpha}, \tau_{\alpha}, X_{\alpha}\right),\left(U_{\alpha}, \tau_{\alpha}\right)$ or just $\tau_{\alpha}$ a trivialization of $\pi^{-1}\left(U_{\alpha}\right)$ and $g_{\alpha \beta}$ a transition map. Note that $g_{\alpha \alpha}(z)=\mathbf{1}_{X_{\alpha}}$ for all $z \in U_{\alpha}, g_{\alpha \beta} g_{\beta \gamma}=g_{\alpha \gamma}$ on $U_{\alpha \beta \gamma}:=U_{\alpha} \cap U_{\beta} \cap U_{\gamma} \neq \emptyset$, and $g_{\alpha \beta}(z)^{-1}=g_{\beta \alpha}(z)$ for all $z \in U_{\alpha \beta}$. For convenience, we often write $\mathcal{E}$ in place of $(\mathcal{E}, \pi, \Omega)$.

If $X$ is a Banach space and $\Omega$ is a complex manifold, the triple $(\Omega \times X, \pi, \Omega)$, where $\pi$ is the canonical projection from $\Omega \times X$ onto $\Omega$, together with the covering trivialisation $\left(\mathbf{1}_{\Omega \times X}: \Omega \times X \rightarrow \Omega \times X\right)$ is called the trivial bundle. If $\mathcal{E}$ is a holomorphic vector bundle and $(U, \tau, X)$ is a trivialisation of $\pi^{-1}(U)$ then $\mathcal{E}_{U}:=\left(\pi^{-1}(U),\left.\pi\right|_{\pi^{-1}(U)}, U\right)$ is a trivial bundle with covering trivialisation $(U, \tau, X)$.

A holomorphic section of the holomorphic vector bundle $(\mathcal{E}, \pi, \Omega)$ is a holomorphic mapping $f: \Omega \rightarrow \mathcal{E}$ such that $\pi \circ f=\mathbf{1}_{\Omega}$. We let $\Gamma(\mathcal{E})$ denote the set of all holomorphic sections of $\mathcal{E}$. For any complex manifold $\Omega$ and any Banach space $X, \Gamma(\Omega \times X) \simeq \mathcal{H}(\Omega, X)$.

In proving the main result in this section we require the following important theorem of Lempert [8].

Theorem 1. Let $Z$ be a Banach space with an unconditional basis, $\Omega \subset Z$ pseudo-convex open, $\mathcal{E} \rightarrow \Omega$ a holomorphic Banach vector bundle, then the sheaf coholomogy groups $H^{q}(\Omega, \mathcal{E})$ vanish for all $q \geq 1$.

Let $\left(U_{\alpha}\right)_{\alpha \in \Gamma}$ be an open covering of $\Omega$. A Cousin data for $\left(U_{\alpha}\right)_{\alpha \in \Gamma}$ is a collection of functions $f_{\alpha \beta} \in$ $\mathcal{H}\left(U_{\alpha \beta}, \mathcal{E}\right)$ satisfying $f_{\alpha \beta}+f_{\beta \alpha}=0$ on $U_{\alpha \beta}:=U_{\alpha} \cap U_{\beta} \neq \emptyset$, and $f_{\alpha \beta}+f_{\beta \gamma}+f_{\gamma \alpha}=0$ on $U_{\alpha \beta \gamma}:=$ $U_{\alpha} \cap U_{\beta} \cap U_{\gamma}$ whenever $U_{\alpha \beta \gamma} \neq \emptyset$. The additive Cousin problem consists in finding $f_{\alpha} \in \mathcal{H}\left(U_{\alpha}, \mathcal{E}\right)$, for all $\alpha$, such that

$$
\left.f_{\alpha}\right|_{U_{\alpha \beta}}-\left.f_{\beta}\right|_{U_{\alpha \beta}}=f_{\alpha \beta}
$$

whenever $U_{\alpha \beta} \neq \emptyset$. Since the Cousin data form a 1-cocycle, when $q=1$ Theorem 1 implies the following result.

Corollary 1. Let $Z$ be a Banach space with an unconditional basis, $\Omega$ be a pseudo-convex open subset of $Z$, and $(\mathcal{E}, \pi, \Omega)$ a holomorphic Banach vector bundle. If $\left(U_{\alpha}\right)_{\alpha \in \Gamma}$ is an open cover of $\Omega$ and $f_{\alpha \beta} \in$ $\mathcal{H}\left(U_{\alpha \beta}, \mathcal{E}\right)$ is a Cousin data then the corresponding Cousin problem is solvable.

Example 1. If $(\mathcal{E}, \pi, \Omega)$ is a holomorphic vector bundle we let $\mathcal{L}(\mathcal{E})=\bigcup_{z \in \Omega} \mathcal{L}\left(\mathcal{E}_{z}\right)$ and let $\theta\left(T_{z}\right)=z$ for all $T_{z} \in \mathcal{L}\left(\mathcal{E}_{z}\right)$. Then $\theta: \mathcal{L}(\mathcal{E}) \rightarrow \Omega$ is surjective and $\theta^{-1}(\{z\})=\mathcal{L}(\mathcal{E})_{z}=\mathcal{L}\left(\mathcal{E}_{z}\right)$. We endow $\mathcal{L}(\mathcal{E})_{z}$ with the Banach space structure from $\mathcal{L}\left(\mathcal{E}_{z}\right)$. Let $\left\{\tau_{\alpha}: \pi^{-1}\left(U_{\alpha}\right) \rightarrow U_{\alpha} \times X_{\alpha}\right\}_{\alpha \in \Lambda}$ be a trivialising cover for $\mathcal{E}$. For $z \in U_{\alpha}$ and $T_{z} \in \mathcal{L}\left(\mathcal{E}_{z}\right)$ let

$$
\hat{\tau}_{\alpha}\left(T_{z}\right)=\left(z, \tau_{\alpha, z} \circ T_{z} \circ \tau_{\alpha, z}^{-1}\right)
$$

Then $\hat{\tau}_{\alpha}: \theta^{-1}\left(U_{\alpha}\right) \rightarrow U_{\alpha} \times \mathcal{L}\left(X_{\alpha}\right)$ is a bijective mapping and $\hat{\tau}_{\alpha, z}: \mathcal{L}(\mathcal{E})_{z} \rightarrow \mathcal{L}\left(X_{\alpha}\right)$ is a continuous linear mapping for all $z \in U_{\alpha}$. If

\footnotetext{
${ }^{1}$ Here and elsewhere we identify, when necessary, $\{z\} \times X_{\alpha}$ and $X_{\alpha}$.
} 


$$
\hat{\tau}_{\alpha \beta}:=\hat{\tau}_{\alpha} \circ \hat{\tau}_{\beta}^{-1}: U_{\alpha \beta} \times \mathcal{L}\left(X_{\beta}\right) \longrightarrow U_{\alpha \beta} \times \mathcal{L}\left(X_{\alpha}\right)
$$

then, for $z \in U_{\alpha \beta}$ and $T \in \mathcal{L}\left(X_{\beta}\right)$, we have

$$
\hat{\tau}_{\alpha \beta}(z, T)=\left(z, g_{\alpha \beta}(z) \circ T \circ g_{\beta \alpha}(z)\right)
$$

where, as previously, $\rho_{\alpha \beta}$, and the transition mappings $g_{\alpha \beta}$ are defined by

$$
\rho_{\alpha \beta}(z, x):=\tau_{\alpha} \circ \tau_{\beta}^{-1}(z, x)=:\left(z, g_{\alpha \beta}(z) x\right)
$$

for $z \in U_{\alpha \beta}$ and $x \in X_{\beta}$. This implies that $\hat{\tau}_{\alpha \beta}$ is biholomorphic for all $\alpha, \beta \in \Lambda$ whenever $U_{\alpha \beta} \neq \emptyset$. The bijective mappings $\left(\hat{\tau}_{\alpha}\right)_{\alpha \in \Lambda}$ can now be used with (2) to define a unique complex manifold structure on $\mathcal{L}(\mathcal{E})$ such that $\hat{\tau}_{\alpha}: \theta^{-1}\left(U_{\alpha}\right) \rightarrow U_{\alpha} \times \mathcal{L}\left(X_{\alpha}\right)$ is biholomorphic for all $\alpha$ and such that $(\mathcal{L}(\mathcal{E}), \theta, \Omega)$ is a holomorphic vector bundle with trivialising cover $\left(U_{\alpha}, \hat{\tau}_{\alpha}\right)_{\alpha \in \Lambda}$. This bundle has transition maps $\hat{g}_{\alpha \beta} \in$ $\mathcal{H}\left(U_{\alpha \beta}, \mathcal{L}\left(\mathcal{L}\left(X_{\beta}\right), \mathcal{L}\left(X_{\alpha}\right)\right)\right)$ where

$$
\left[\hat{g}_{\alpha \beta}(z)\right](T)=g_{\beta \alpha}(z) \circ T \circ g_{\alpha \beta}(z)
$$

for $z \in U_{\alpha \beta}$ and $T \in \mathcal{L}\left(X_{\beta}\right)$.

A sub-bundle of $(\mathcal{E}, \pi, \Omega)$ is a bundle $(\mathcal{F}, \eta, \Omega)$ where $\mathcal{F}$ is a subset of $\mathcal{E}, \eta=\left.\pi\right|_{\mathcal{F}}, \mathcal{F}_{z}$ is a closed subspace of $\mathcal{E}_{z}$ for all $z \in \Omega$ and the following condition holds:

There exists a trivialising cover $\left\{\tau_{\alpha}: \pi^{-1}\left(U_{\alpha}\right) \rightarrow U_{\alpha} \times X_{\alpha}\right\}_{\alpha \in \Lambda}$ for $\mathcal{E}$, and a collection of Banach spaces $\left(Y_{\alpha}\right)_{\alpha \in \Lambda}, Y_{\alpha} \subset X_{\alpha}$, such that $\left\{\left.\tau_{\alpha}\right|_{\eta^{-1}\left(U_{\alpha}\right)}: \eta^{-1}\left(U_{\alpha}\right) \rightarrow U_{\alpha} \times Y_{\alpha}\right\}_{\alpha \in \Lambda}$ is a trivialising cover for $\mathcal{F}$.

Note that a sub-bundle is defined locally, that is given a bundle $(\mathcal{E}, \pi, \Omega)$ and an open cover of $\Omega,\left(U_{\alpha}\right)_{\alpha}$, and for each $\alpha$ a sub-bundle $\mathcal{F}_{\alpha}$ of $\mathcal{E}_{U_{\alpha}}$, then there exists a unique sub-bundle $\mathcal{F}$ of $\mathcal{E}$ such that $\mathcal{F}_{U_{\alpha}}=\mathcal{F}_{\alpha}$.

This means that we may and do identify $Y_{\alpha}$ with a subspace of $X_{\alpha}$ and, moreover, that $\left[g_{\alpha \beta}(z)\right] Y_{\beta}=Y_{\alpha}$ for the transition functions $g_{\alpha \beta}$ where $z \in U_{\alpha \beta}$ and $\alpha, \beta \in \Lambda$. If each $Y_{\alpha}$ is a complemented subspace of $X_{\alpha}$ the sub-bundle is called a direct sub-bundle.

Sub-bundles can also be characterised by using transition functions. Suppose we are given a trivialising cover $\left\{\tau_{\alpha}: \pi^{-1}\left(U_{\alpha}\right) \rightarrow U_{\alpha} \times X_{\alpha}\right\}_{\alpha \in \Lambda}$ for $\mathcal{E}$ with transition functions $\left(g_{\alpha \beta}\right)_{\alpha, \beta \in \Lambda}$, and a collection of Banach spaces $\left(Y_{\alpha}\right)_{\alpha \in \Lambda}, Y_{\alpha} \subset X_{\alpha}$, such that $\left[g_{\alpha \beta}(z)\right] Y_{\beta} \subset Y_{\alpha}$ for all $\alpha, \beta \in \Lambda$ and all $z \in U_{\alpha} \cap U_{\beta}$. Since $g_{\alpha \beta}(z)^{-1}=g_{\beta \alpha}(z)$ this implies

$$
\left[g_{\alpha \beta}(z)\right] Y_{\beta}=Y_{\alpha}
$$

for all $z \in U_{\alpha \beta}$. Let $\mathcal{F}=\cup_{\alpha \in \Lambda} \pi^{-1}\left(U_{\alpha} \times Y_{\alpha}\right), \eta=\left.\pi\right|_{\mathcal{F}}$ and $\varphi_{\alpha}=\left.\tau_{\alpha}\right|_{\eta^{-1}\left(U_{\alpha}\right)}$ for all $\alpha \in \Lambda$. Then $\varphi_{\alpha, z}: \eta^{-1}(\{z\})=\mathcal{F}_{z} \rightarrow\{z\} \times Y_{\alpha}$ is bijective and the Banach space $\mathcal{E}_{z}$ induces on $\mathcal{F}_{z}$ a Banach space structure. Since each $\varphi_{\alpha}$ is the restriction of a bijective mapping it also is bijective onto its image and as $\varphi_{\alpha \beta}:=\varphi_{\alpha} \circ \varphi_{\beta}^{-1}(z, y)=\left(z, g_{\alpha \beta}(z) y\right)$ for all $(z, y) \in U_{\alpha \beta} \times Y_{\beta}$ we see, by (3), that $(\mathcal{F}, \eta, \Omega)$ is a holomorphic vector bundle with trivialising cover $\left\{\varphi_{\alpha}: \eta^{-1}\left(U_{\alpha}\right) \rightarrow U_{\alpha} \times Y_{\alpha}\right\}_{\alpha \in \Lambda}$. Since $\varphi_{\alpha}=$ $\left.\tau_{\alpha}\right|_{\eta^{-1}\left(U_{\alpha}\right)}, \mathcal{F}$ is a sub-bundle of $\mathcal{E}$.

Example 2. Let $(\mathcal{F}, \eta, \Omega)$ be a sub-bundle of the holomorphic vector bundle $(\mathcal{E}, \pi, \Omega)$. By definition we can find a trivialising cover for $\pi,\left\{\tau_{\alpha}: \pi^{-1}\left(U_{\alpha}\right) \rightarrow U_{\alpha} \times X_{\alpha}\right\}_{\alpha \in \Lambda}$ and a collection of Banach spaces $\left(Y_{\alpha}\right)_{\alpha \in \Lambda}, Y_{\alpha} \subset X_{\alpha}$, such that $\left\{\left.\tau_{\alpha}\right|_{\eta^{-1}\left(U_{\alpha}\right)}: \eta^{-1}\left(U_{\alpha}\right) \rightarrow U_{\alpha} \times Y_{\alpha}\right\}$ is a trivialising cover for $\eta$. Let $(\mathcal{L}(\mathcal{E}), \theta, \Omega)$ denote the holomorphic vector bundle with trivialising cover $\left\{\hat{\tau}_{\alpha}: \theta^{-1}\left(U_{\alpha}\right) \rightarrow U_{\alpha} \times\right.$ $\left.\mathcal{L}\left(X_{\alpha}\right)\right\}_{\alpha \in \Lambda}$ constructed in Example 1 .

For each $\alpha \in \Lambda$ let 


$$
Z_{\alpha}:=\left\{T \in \mathcal{L}\left(X_{\alpha}\right): T\left(X_{\alpha}\right) \subset Y_{\alpha}, T\left(Y_{\alpha}\right)=0\right\} .
$$

For $\alpha, \beta \in \Lambda, z \in U_{\alpha \beta}$ and $T \in Z_{\beta}$ we have

$$
\begin{aligned}
{\left[\hat{g}_{\alpha \beta}(z)(T)\right]\left(X_{\alpha}\right) } & \subset g_{\alpha \beta}(z) \circ T\left(g_{\beta \alpha}(z) X_{\alpha}\right) \\
& \subset g_{\alpha \beta}(z) \circ T\left(X_{\beta}\right) \\
& \subset g_{\alpha \beta}(z)\left(Y_{\beta}\right) \\
& \subset Y_{\alpha}
\end{aligned}
$$

and

$$
\left[\hat{g}_{\alpha \beta}(z)(T)\right]\left(Y_{\alpha}\right) \subset g_{\alpha \beta}(z)\left(T\left(Y_{\beta}\right)\right)=\{0\} .
$$

Hence $\hat{g}_{\alpha \beta}(z)\left(Z_{\beta}\right) \subset Z_{\alpha}$ for all $z \in U_{\alpha \beta}$. This implies, following our discussion above, that $\mathcal{L}(\mathcal{E} \odot \mathcal{F}):=$ $\cup_{\alpha \in \Lambda} \hat{\tau}_{\alpha}^{-1}\left(U_{\alpha} \times Z_{\alpha}\right)$ can be endowed with the structure of a sub-bundle of $\mathcal{L}(\mathcal{E})$.

An endomorphism of the holomorphic vector bundle $(\mathcal{E}, \pi, \Omega)$ is a holomorphic mapping $f: \mathcal{E} \rightarrow \mathcal{E}$ such that $f \circ \pi=\pi, f_{z}:=\left.f\right|_{\mathcal{E}_{z}}$ is a continuous linear mapping for all $z \in \Omega$, and the mapping

$$
z \in U \longrightarrow \tau_{z} \circ f_{z} \circ \tau_{z}^{-1} \in \mathcal{L}(X)
$$

is holomorphic for any trivialising map $\tau: \pi^{-1}(U) \rightarrow U \times X$. We denote by $\mathcal{M}(\mathcal{E})$ the set of all endomorphisms of $\mathcal{E}$. If $f_{z}^{2}=f_{z}$ for all $z \in \Omega$ we call $f$ a projection.

Using the notation of Examples 1 and 2 we see that the mapping

$$
\theta: \mathcal{M}(\mathcal{E}) \longrightarrow \Gamma(\mathcal{L}(\mathcal{E})), \quad[\theta(A)](z):=\left.A\right|_{\mathcal{E}_{z}}
$$

is bijective and, moreover, if $\mathcal{F}$ is a sub-bundle of $\mathcal{E}$ then

$$
A(\mathcal{E}) \subset \mathcal{F} \Longleftrightarrow[\theta(A)(z)] \mathcal{E}_{z} \subset \mathcal{F}_{z} \text { for all } z \in \Omega
$$

and

$$
A(\mathcal{F})=\{0\} \Longleftrightarrow[\theta(A)(z)] \mathcal{F}_{z}=\{0\} \text { for all } z \in \Omega .
$$

Clearly $A \in \mathcal{M}(\mathcal{E})$ is a projection if and only if $[\theta(A)](z)$ is a (linear) projection for all $z \in \Omega$. For the trivial bundle, $\mathcal{M}(\Omega \times X) \simeq \mathcal{H}(\Omega, \mathcal{L}(X))$.

Proposition 2. Let $\Omega$ be a pseudo-convex open subset of a Banach space with an unconditional basis. If $\mathcal{F}:=(\mathcal{F}, \eta, \Omega)$ is a sub-bundle of the holomorphic vector bundle $(\mathcal{E}, \pi, \Omega)$ then $\mathcal{F}$ is a direct sub-bundle if and only if there exists a projection $p \in \mathcal{M}(\mathcal{E})$ such that $p(\mathcal{E})=\mathcal{F}$.

Proof. We first suppose that $\mathcal{F}$ is a direct sub-bundle of $\mathcal{E}$. Let $\left\{\tau_{\alpha}: \pi^{-1}\left(U_{\alpha}\right) \rightarrow U_{\alpha} \times X_{\alpha}\right\}_{\alpha \in \Lambda}$ denote a trivialising cover for $\mathcal{E}$ such that $\left\{\left.\tau_{\alpha}\right|_{\eta^{-1}\left(U_{\alpha}\right)}: \eta^{-1}\left(U_{\alpha}\right) \rightarrow U_{\alpha} \times Y_{\alpha}\right\}$ is a trivialising cover for $\mathcal{F}$. By our hypothesis $Y_{\alpha}$ is a complemented subspace of $X_{\alpha}$ and we let $P_{\alpha} \in \mathcal{L}\left(X_{\alpha}\right)$ denote a continuous projection onto $Y_{\alpha}$ for each $\alpha \in \Lambda$. For each $\alpha$ let $\mathcal{E}_{\alpha}$ denote the holomorphic vector bundle $\left(\pi^{-1}\left(U_{\alpha}\right),\left.\pi\right|_{\pi^{-1}\left(U_{\alpha}\right)}, U_{\alpha}\right)$ with trivialising cover $\left(U_{\alpha}, \tau_{\alpha}, X_{\alpha}\right)$. Then $\mathcal{F}_{\alpha}:=\left(\eta^{-1}\left(U_{\alpha}\right),\left.\eta\right|_{\eta^{-1}\left(U_{\alpha}\right)}, U_{\alpha}\right)$ with trivialising cover $\left(U_{\alpha},\left.\tau_{\alpha}\right|_{\eta^{-1}\left(U_{\alpha}\right)}, Y_{\alpha}\right)$ is a direct sub-bundle of $\mathcal{E}_{\alpha}$. We define $f_{\alpha}: \mathcal{E}_{\alpha} \rightarrow \mathcal{E}_{\alpha}$ as follows: if $z \in U_{\alpha}$ let $\left.f_{\alpha}\right|_{\mathcal{E}_{z}}=: f_{\alpha, z}$ where

$$
f_{\alpha, z}(\xi)=\tau_{\alpha, z}^{-1} \circ P_{\alpha} \circ \tau_{\alpha, z}(\xi)
$$


for all $\xi \in \mathcal{E}_{z}$. Then $f_{\alpha, z} \in \mathcal{L}\left(\mathcal{E}_{z}\right)$ is a projection with $f_{\alpha, z}\left(\mathcal{E}_{z}\right)=\mathcal{F}_{z}$ for all $z \in U_{\alpha}$. Since $\tau_{\alpha, z} \circ f_{\alpha} \circ \tau_{\alpha, z}^{-1}=$ $P_{\alpha}, f_{\alpha} \in \mathcal{M}\left(\mathcal{E}_{\alpha}\right)$ and $f_{\alpha}\left(\mathcal{E}_{\alpha}\right)=\mathcal{F}_{\alpha}$.

If $\alpha, \beta \in \Lambda$ and $U_{\alpha \beta} \neq \emptyset$ let $f_{\alpha \beta}=\left.f_{\alpha}\right|_{\mathcal{E}_{\alpha \beta}}-\left.f_{\beta}\right|_{\mathcal{E}_{\alpha \beta}}$. Then $f_{\alpha \beta} \in \mathcal{M}\left(\mathcal{E}_{\alpha \beta}\right)$ and $f_{\alpha \beta}\left(\mathcal{E}_{\alpha \beta}\right) \subset \mathcal{F}_{\alpha \beta}$. Since $f_{\alpha}(\xi)=f_{\beta}(\xi)=\xi$ for all $z \in U_{\alpha \beta}$ and all $\xi \in \mathcal{F}_{z}, f_{\alpha \beta}\left(\mathcal{F}_{\alpha \beta}\right)=\{0\}$. By (5) we can identify $f_{\alpha \beta}$ with $g_{\alpha \beta} \in \Gamma\left(\mathcal{L}\left(\mathcal{E}_{\alpha \beta}\right)\right)$ and, by Example 2 and (6) and (7), $g_{\alpha \beta} \in \Gamma\left(\mathcal{L}\left(\mathcal{E}_{\alpha \beta} \odot \mathcal{F}_{\alpha \beta}\right)\right)$. Since $\left(g_{\alpha \beta}\right)_{\alpha, \beta \in \Lambda}$ forms a 1-cocycle in the sheaf of $\mathcal{L}(\mathcal{E} \odot \mathcal{F})$-valued holomorphic germs on $\Omega$, Corollary 1 implies that there exist, for all $\alpha \in \Lambda, g_{\alpha} \in \Gamma\left(\mathcal{L}\left(\mathcal{E}_{\alpha} \odot \mathcal{F}_{\alpha}\right)\right)$ such that

$$
\left.g_{\alpha}\right|_{U_{\alpha \beta}}-\left.g_{\beta}\right|_{U_{\alpha \beta}}=g_{\alpha \beta} .
$$

By (5) each $g_{\alpha}$ can be identified with $h_{\alpha} \in \mathcal{M}\left(\mathcal{E}_{\alpha}\right)$, satisfying $h_{\alpha}\left(\mathcal{E}_{\alpha}\right) \subset \mathcal{F}_{\alpha}$ and $h_{\alpha}\left(\mathcal{F}_{\alpha}\right)=0$ and, by (8),

for all $\alpha, \beta \in \Lambda$ whenever $U_{\alpha \beta} \neq \emptyset$. Hence

$$
\left.h_{\alpha}\right|_{\mathcal{E}_{\alpha \beta}}-\left.h_{\beta}\right|_{\mathcal{E}_{\alpha \beta}}=\left.f_{\alpha}\right|_{\mathcal{E}_{\alpha \beta}}-\left.f_{\beta}\right|_{\mathcal{E}_{\alpha \beta}}
$$

whenever $U_{\alpha \beta} \neq \emptyset$ and the mapping

$$
\left.\left(f_{\alpha}-h_{\alpha}\right)\right|_{\mathcal{E}_{\alpha \beta}}=\left.\left(f_{\beta}-h_{\beta}\right)\right|_{\mathcal{E}_{\alpha \beta}}
$$

$$
p(\xi):=f_{\alpha}(\xi)-h_{\alpha}(\xi)
$$

for all $\xi \in \pi^{-1}\left(U_{\alpha}\right)$ is well defined on $\mathcal{E}$ and belongs to $\mathcal{M}(\mathcal{E})$. Since $f_{\alpha}$ and $h_{\alpha}$ both map $\mathcal{E}_{\alpha}$ into $\mathcal{F}_{\alpha}$ for all $\alpha \in \Lambda$ it follows that $p(\mathcal{E}) \subset \mathcal{F}$ and as $f_{\alpha}\left(\mathcal{F}_{\alpha}\right)=\mathcal{F}_{\alpha}$ and $h_{\alpha}\left(\mathcal{F}_{\alpha}\right)=\{0\}$ this implies $p(\mathcal{E})=\mathcal{F}$. If $z \in U_{\alpha}$ and $\xi \in \mathcal{E}_{z}$ then $f_{\alpha, z}\left(h_{\alpha, z}(\xi)\right)=h_{\alpha, z}(\xi), h_{\alpha, z}\left(f_{\alpha, z}(\xi)\right)=0$, and $h_{\alpha, z}\left(h_{\alpha, z}(\xi)\right)=0$. Hence

$$
\begin{aligned}
p(p(\xi)) & =p\left(f_{\alpha, z}(\xi)-h_{\alpha, z}(\xi)\right) \\
& =f_{\alpha, z}^{2}(\xi)-f_{\alpha, z}\left(h_{\alpha, z}(\xi)\right)-h_{\alpha, z}\left(f_{\alpha, z}(\xi)\right)+h_{\alpha, z}\left(h_{\alpha, z}(\xi)\right) \\
& =f_{\alpha, z}(\xi)-h_{\alpha, z}(\xi) \\
& =p(\xi) .
\end{aligned}
$$

This completes the proof in one direction.

Since the converse is a local result we may suppose that $\mathcal{E}$ is the trivial bundle, $\Omega \times X$, that $p \in$ $\mathcal{H}(\Omega, \mathcal{L}(X))$ and $p(z)$ is a projection for all $z \in \Omega$. We must show that $\mathcal{F}:=\{(z, x): x=p(z) x\}$ is a direct sub-bundle of $\mathcal{E}$. Fix $w \in \Omega$, and let $X_{0}:=p(w) X, X_{1}:=\left(\mathbf{1}_{X}-p(w)\right) X$. For $z \in \Omega$ let

$$
A(z):=p(z) p(w)+\left(\mathbf{1}_{X}-p(z)\right)\left(\mathbf{1}_{X}-p(w)\right) .
$$

Since $A(w)=\mathbf{1}_{X}$ we can choose a neighbourhood of $w, V_{w}$, such that $A(z)$ is invertible on $V_{w}$. Then

and

$$
A(z)\left(X_{0}\right)=p(z) p(w) X \subset p(z) X
$$

$$
\begin{aligned}
A(z)\left(X_{1}\right) & =\left(\mathbf{1}_{X}-p(z)\right)\left(\mathbf{1}_{X}-p(w)\right) X \\
& \subset\left(\mathbf{1}_{X}-p(z)\right) X .
\end{aligned}
$$

Since $A(z)$ is invertible on $V_{w}$ we have $A(z)\left(X_{0}+X_{1}\right)=X$, hence $A(z)\left(X_{0}\right)=p(z) X$ and $A(z)\left(X_{1}\right)=$ $\left(\mathbf{1}_{X}-p(z)\right) X$. If $B(z)$ denotes the inverse of $A(z)$ then $X_{0}=B(z)(p(z) X)$ for all $z \in V_{w}$ and the mapping

provides the required trivialization. This completes the proof.

$$
V_{w} \times X \rightarrow V_{w} \times X:(z, x) \rightarrow(z, B(z) x)
$$

Note that we did not require pseudo-convexity or Corollary 1 for the second half of the proof. 


\section{Generalised Inverses}

In this section we consider the following question: if $f \in \mathcal{H}(\Omega, \mathcal{L}(X, Y))$ and $f(z)$ has a generalised inverse at all points in $\Omega$, does $f$ have a holomorphic generalised inverse?

Definition 3. Let $f \in \mathcal{H}(\Omega, \mathcal{L}(X, Y))$, where $X$ and $Y$ are Banach spaces and $\Omega$ is an open subset of a Banach space. A mapping $g \in \mathcal{H}(\Omega, \mathcal{L}(Y, X))$ is called a holomorphic generalised inverse for $f$ if $g(z)$ is a generalised inverse for $f(z)$ for all $z \in \Omega$.

The following example shows that a holomorphic generalised inverse need not always exist.

Example 3. If $h(z)=z \mathbf{1}_{H}$, where $H$ is a one dimensional Hilbert space, then $h \in \mathcal{H}(\mathbb{C}, \mathcal{L}(H))$. If $z \neq 0, f(z)$ is invertible and we have a unique generalised inverse $g(z):=(f(z))^{-1}=z^{-1} \mathbf{1}_{H}$. Since $\lim _{z \rightarrow 0} g(z)$ does not exist $f$ does not have a holomorphic generalised inverse.

Proposition 3. Let $f \in \mathcal{H}(\Omega, \mathcal{L}(X, Y))$, where $X$ and $Y$ are Banach spaces and $\Omega$ is an open subset of a Banach space. Then $f$ has a holomorphic generalised inverse if and only if there exist $P \in \mathcal{H}(\Omega, \mathcal{L}(X))$ and $Q \in \mathcal{H}(\Omega, \mathcal{L}(Y))$ such that $P(z)$ is a continuous projection onto $\operatorname{ker}(f(z))$ and $Q(z)$ is a continuous projection onto $\operatorname{im}(f(z))$ for all $z \in \Omega$.

PROOF. If $g$ is a holomorphic generalised inverse for $f$ then the mappings $P$ and $Q$, defined by letting $P(z):=g(z) \circ f(z)$ and $Q(z):=f(z) \circ g(z)$, are the required projection-valued holomorphic mappings.

Conversely, suppose we are given the projection-valued holomorphic mappings $P$ and $Q$. For convenience let $P^{*}(z)=\mathbf{1}_{X}-P(z)$ and let $I_{z}$ denote the natural injection from $P^{*}(z) X$ into $X$ for all $z \in \Omega$. Let

$$
g(z):=I_{z} \circ\left(f^{*}(z)\right)^{-1} \circ Q(z)
$$

where $f^{*}(z)=\left.f(z)\right|_{P^{*}(z) X}$. The linear result in the second section shows that $g(z)$ is a generalised inverse for $f(z)$ for all $z \in \Omega$.

To show that $g$ is holomorphic we fix $w \in \Omega$ and choose $\epsilon>0$ such that $W:=\{z:\|z-w\|<\epsilon\} \subset \Omega$, $\|P(z)-P(w)\|<1$ and $\|Q(z)-Q(w)\|<1$ for all $z \in W$. Let $U(z)=\mathbf{1}_{X}+P(z)-P(w)=$ $\mathbf{1}_{X}-P^{*}(z)+P^{*}(w)$ and $V(z)=\mathbf{1}_{Y}-Q(z)+Q(w)=\mathbf{1}_{Y}+Q^{*}(z)-Q^{*}(w)$ for all $z \in W$. By Lemma 1 , $U \in \mathcal{H}(W, G L(X)), V \in \mathcal{H}(W, G L(Y)), U(z)\left(P^{*}(z) X\right)=P^{*}(w) X$ and $V(z)(Q(z) Y)=Q(w) Y$ for all $z \in W$. We have

$$
\begin{aligned}
g(z): & =\left(I_{w} \circ U(z)^{-1}\right) \circ\left(U(z) \circ\left(f^{*}(z)\right)^{-1} \circ V(z)^{-1}\right) \circ(V(z) \circ Q(z)) \\
& =\left(I_{w} \circ U(z)^{-1}\right) \circ\left(V(z) \circ f(z) \circ U(z)^{-1}\right)^{-1} \circ(V(z) \circ Q(z)) .
\end{aligned}
$$

Since $V(z) \circ Q(z)=Q(w) \circ Q(z)$ for all $z \in W$ the mapping $z \rightarrow V(z) \circ Q(z)$ lies in $\mathcal{H}(W, \mathcal{L}(Y, Q(w) Y)$. By Lemma 1, the mapping $z \in W \rightarrow I_{w} \circ U(z)^{-1}$ belongs to $\mathcal{H}\left(W, \mathcal{L}\left(P^{*}(w) X, X\right)\right.$. It remains to show that the mapping

$$
z \longrightarrow k(z):=\left(V(z) \circ f(z) \circ U(z)^{-1}\right)^{-1}
$$

lies in $\mathcal{H}\left(W, \mathcal{L}\left(Q(w) Y, P^{*}(w) X\right)\right)$. By construction the mapping

$$
z \longrightarrow k^{*}(z):=V(z) \circ f(z) \circ U(z)^{-1}
$$

lies in $\mathcal{H}\left(\Omega, G L\left(P^{*}(w) X, Q(w) Y\right)\right)$ and, as $k(z)=\left(k^{*}(z)\right)^{-1}$, this proves that $k$ is holomorphic. This completes the proof.

We now present the main result in this article. Note that for $z \in \Omega, \operatorname{ker}(f(z))$ is the kernel of a linear operator while $\operatorname{ker}(f)$ is a holomorphic vector bundle.

Theorem 2. Let $\Omega$ be a pseudo-convex open subset of a Banach space with an unconditional basis and let $X$ and $Y$ be Banach spaces. If $f \in \mathcal{H}(\Omega, \mathcal{L}(X, Y))$ has a generalised inverse for each $z \in \Omega$, then the following conditions are equivalent: 
(1) $f$ has a holomorphic generalised inverse on $\Omega$,

(2) There exist holomorphic projections $P \in \mathcal{H}(\Omega, \mathcal{L}(X)) \simeq \mathcal{M}(\Omega \times X)$ onto $\operatorname{ker}(f):=\{(z, x)$ : $z \in \Omega, x \in X, f(z) x=0\}$ and $Q \in \mathcal{H}(\Omega, \mathcal{L}(Y)) \simeq \mathcal{M}(\Omega \times Y)$ onto $\operatorname{im}(f):=\{(z, y):$ $z \in \Omega, y \in Y, y=f(z) x$ for some $x \in X\}$,

(3) $\operatorname{ker}(f)$ and $\operatorname{im}(f)$ are direct sub-bundles of the trivial bundles $\Omega \times X$ and $\Omega \times Y$ respectively,

(4) For every $w \in \Omega$ there exist a neighbourhood $V_{w}$ of $w$ and closed subspaces $X_{w} \subset X$ and $Y_{w} \subset Y$ such that for all $z \in V_{w}, \operatorname{ker}(f(z)) \oplus X_{w}=X$ and $\operatorname{im}(f(z)) \oplus Y_{w}=Y$.

Proof. By Proposition 3, (1) and (2) are equivalent. By Proposition 2, (2) and (3) are equivalent. By the definition of sub-bundle, (3) implies (4), and it remains to show that (4) implies (3).

Since the result is local we fix $w \in \Omega$ and show that (3) holds on a neighbourhood $V_{w}$ of $w$. If $z \in V_{w}$, $x \in X$ and $y \in Y_{w}$ let $g(z)(x+y)=f(z) x+y$. Then $g \in \mathcal{H}\left(V_{w}, \mathcal{L}\left(X+Y_{w}, Y\right)\right)$,

$$
\operatorname{ker}(g(z))=\operatorname{ker}(f(z))+\{0\} \quad \text { and } \quad \operatorname{im}(g(z))=\operatorname{im}(f(z))+Y_{w}=Y
$$

for all $z \in V_{w}$. Hence $g$ is surjective with complemented kernel for all $z \in V_{w}$. By the proof of Proposition 1 (see also Theorem 4 in [4]), $\operatorname{ker}(g)=\left\{(z, x, y) \in V_{w} \times\left(X+Y_{w}\right): f(z) x=0, y=0\right\}$ is a direct holomorphic sub-bundle of the trivial bundle $V_{w} \times\left(X+Y_{w}\right)$. Since $\operatorname{ker}\left(\left.f\right|_{V_{w}}\right) \simeq \operatorname{ker}(g) \subset V_{w} \times(X+$ $\{0\}) \simeq V_{w} \times X$ this implies $\operatorname{ker}\left(\left.f\right|_{V_{w}}\right)$ is a direct sub-bundle of the trivial bundle $V_{w} \times X$.

By Proposition 2 there exist a holomorphic projection $p \in \mathcal{H}\left(V_{w}, \mathcal{L}(X)\right)$ such that $\operatorname{ker}(f(z))=$ $p(z)(X)$ for all $z \in V_{w}$. By Lemma 1 and, if necessary, by restricting ourselves to a smaller neighbourhood of $w$ we have $p(z)(X)=p(w)(X)=: Z_{w}$ for all $z \in V_{w}$. Hence $X=Z_{w} \oplus X_{w}$ and $f(z)(x+y)=f(z)(y)$ for all $z \in V_{w}$, all $x \in Z_{w}=\operatorname{ker}(f(z))$, and all $y \in X_{w}$. If $h(z):=\left.f(z)\right|_{X_{w}}$ then $h \in \mathcal{H}\left(V_{w}, \mathcal{L}\left(X_{w}, Y\right)\right)$, $h(z)$ is injective and $\operatorname{im}(f(z))=\operatorname{im}(h(z))$ is a complemented subspace of $Y$ for all $z \in V_{w}$. By adapting the proof of Proposition 1 in [4] we see that $\operatorname{im}(h)=\operatorname{im}\left(\left.f\right|_{V_{w}}\right)$ is a complemented sub-bundle of the trivial bundle $V_{w} \times Y$. Hence (4) implies (3) and this completes the proof.

Acknowledgement. This work was carried out with the partial support of SFI grant R9317.

\section{References}

[1] Allan, G. R., (1967). On one-sided inverses in Banach algebras of holomorphic vector-valued functions, $J$. London Math. Soc., 42, 463-470.

[2] Atkinson, F. V., (1953). On relatively regular operators, Acta Sc. Math. Szeged., 15, 38-56.

[3] Dineen, S., (1999). Complex Analysis on Infinite Dimensional Spaces, Springer-Verlag Monographs in Mathematics.

[4] Dineen, S., Patyi, I. And Venkova, M., (2006). Inverses Depending Holomorphically on a Parameter in a Banach Space, J. Funct. Anal., 237, 141-156.

[5] Dineen, S. And Venkova, M., Fredholm-Valued Holomorphic Mappings on a Banach Space, Indag. Math. (to appear).

[6] Gohberg, I., Golberg, S. And KaAshoek, M. A., (1990). Classes of Linear Operators, Vol. 1, Birkhäuser, Basel.

[7] Gramsch, B. And Kaballo, W., (1980). Spectral Theory for Fredholm functions, in Functional Analysis: Surveys and Recent Results II, Proceedings of the Paderborn Conference on Functional Analysis, Ed. K-D. Bierstedt and B. Fuchssteiner, North-Holland Mathematical Studies, 38, 319-342. 
[8] Lempert, L., (2004). Vanishing Cohomology for Holomorphic Vector Bundles in a Banach Setting, Asian J. Math., 8, 1, 65-68.

[9] MujicA, J., (1986). Complex Analysis in Banach Spaces, North-Holland Mathematical Studies, 120.

[10] Murphy, G. J., (1990). $C^{*}$-Algebras and Operator Theory, Academic Press, Inc.

[11] Shubin, M. A., (1979). On Holomorphic Families of Subspaces of a Banach Space, Integral Equations and Operator Theory, 2, 3, 407-420, (Translation from Mat. Issled. (Kishinev) 5, vyp 4 (18), 1970, 153-165.)

[12] Zaidenberg, M. G., Krein, S. G., Kuchment, P. A. and Pankov, A. A., (1975). Banach Bundles and Linear Operators, Russian Math. Surveys, 30, 5, 115-175.

\section{Seán Dineen}

School of Mathematical Sciences,

University College

Dublin,

Dublin 4,

Ireland.

\section{Milena Venkova}

School of Mathematical Sciences, University College

Dublin,

Dublin 4,

Ireland. 